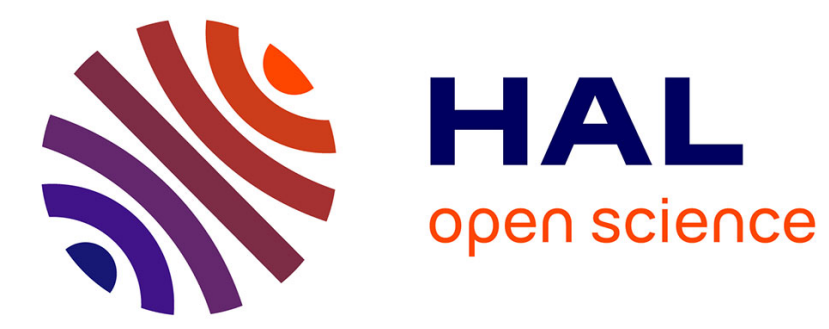

\title{
3D angiographic image segmentation
}

Benoît Naegel, Nicolas Passat, Christian Ronse

\section{To cite this version:}

Benoît Naegel, Nicolas Passat, Christian Ronse. 3D angiographic image segmentation. Mathematical Morphology: From Theory to Applications, pp.375-383, 2010. hal-01694517

\section{HAL Id: hal-01694517 https://hal.univ-reims.fr/hal-01694517}

Submitted on 11 Mar 2018

HAL is a multi-disciplinary open access archive for the deposit and dissemination of scientific research documents, whether they are published or not. The documents may come from teaching and research institutions in France or abroad, or from public or private research centers.
L'archive ouverte pluridisciplinaire HAL, est destinée au dépôt et à la diffusion de documents scientifiques de niveau recherche, publiés ou non, émanant des établissements d'enseignement et de recherche français ou étrangers, des laboratoires publics ou privés. 


\title{
3D angiographic image segmentation
}

\author{
Benoît Naegel, Nicolas Passat, Christian Ronse
}

March 11, 2018

\section{Context}

Medical images provide, at each point of the visualized space, some information related to given physical/chemical properties of tissues. The signal related to specific anatomical structures can be improved by using contrast media. Nevertheless, despite of such image acquisition optimisations, the detection of these structures of interest (i.e., their segmentation) remains very hard to carry out in an automatic and reliable fashion. In particular, due to the variability of the signal and the noise induced by the image acquisition devices, the use of (local) photometric information is actually insufficient to efficiently perform this fundamental task.

The purpose of segmentation is to help the medical expert by enabling an easy detection of specific structures. Consequently, the designed methods have to rely on efficient strategies for extracting anatomical, pathological or functional structures, especially by determining not only what is sought, but also where and how it should be searched. It is then no longer sufficient to deal with the signal of images; it becomes important to also carefully consider their semantics.

When analysing anatomical structures that present a high level of complexity, it is possible to take into account semantic aspects by studying notions such as shape, position, size or orientation, which can be expressed in terms of morphology (set-based operations, relying on structuring elements) and geometry (length, curvature, diameter, etc.). Based on these considerations, some solutions are proposed hereafter, to develop segmentation tools relying on mathematical morphology operators guided by high-level knowledge elements. By focusing on the case of angiographic imaging (i.e., the imaging devoted to vascular structures), this chapter illustrates -non exhaustivelythe way to take into account and formalize anatomical knowledge elements in mathematical morphology. In particular, in the following two sections, two concepts enabling to tackle this problem are described: the anatomical knowledge models, and the hit-ormiss transform. Two examples of segmentation methods based on these concepts are then presented, in order to emphasize the soundness of the proposed approach [11].

\section{Anatomical knowledge modelling}

In order to process spatial information contained in medical images, the use of robust anatomical hypotheses is essential. It is in particular necessary to allow the modelling 
of this knowledge to express both anatomical invariants and inter-individual variability. Indeed, in mathematical morphology, it is important, to be able to adapt the used structuring elements to the objects assumed to be present in the processed image at the considered point. To this end, the structuring element can, for instance, be chosen with respect to a model of the search object, obtained beforehand by a learning process.

A first approach can consist in creating shape models, which describe the general shape of structures of interest. Such models can be defined by computing the mean image of data obtained from the segmentation of a (learning) image database. This mean image of binary functions is then a grey-level function taking its values in the interval $[0,1]$. An example of this kind of models, built from a base composed of 15 segmented images of the portal vein entry, is illustrated in Figure 2(a), and can be expressed as:

$$
\begin{aligned}
\mathcal{M}: & E \\
x & \mapsto \mathcal{M}(x)
\end{aligned}
$$

where $E$ is the space on which the anatomical structure is defined, and $\mathcal{M}(x)$ is the mean presence ratio of the structure at point $x$, with respect to the learning base. The sets $\mathcal{M}^{-1}(\{1\})$ and $\left.\left.\mathcal{M}^{-1}(] 0,1\right]\right)$ constitute a subset and a superset of the considered anatomical structure, respectively. Such a model can lead to the generation of structuring elements which enable the extraction of this anatomical structure in images which do not belong to the learning base, but which are similar. In [12], these elements are made of cylinders and horizontal/vertical concentric discrete circles.

A second approach consists in using simpler morphological hypotheses (for instance a tubular model in the case of vessels) and to focus more accurately on quantitative criteria. This strategy is illustrated by the notion of statistical atlas, which aims at estimating the value of a series of parameters according to the position in the image. The parameters proposed in the atlas presented hereafter, related to the cerebral vascular network, deal with the presence probability, the size and the orientation of vessels. An example of atlas, obtained from a learning database composed of 18 images [14], is illustrated in Figure 2(b), and can be expressed as:

$$
\begin{array}{|cccc}
\mathcal{A}: & E & \rightarrow & {[0,1] \times \mathcal{P}\left(\mathbb{R}^{+}\right) \times \mathcal{P}([0, \pi[\times[0, \pi[)} \\
x & \mapsto & \left(\mathcal{A l}^{d}(x), \mathcal{A}^{t}(x), \mathcal{A}^{o}(x)\right)
\end{array}
$$

where $\mathcal{A}^{d}, \mathcal{A}^{t}$ and $\mathcal{A}^{o}$ represent the fields related to presence probability ${ }^{1}$, size and orientation, respectively (the last two fields are expressed as intervals modelling the inter-individual variability). Such an atlas is meaningful only if it is associated to an anatomical reference which can, for instance, be an image $R: E \rightarrow \mathbb{Z}$ visualizing related anatomical structures. The information provided by $\mathcal{A}(x)$ then correspond to the anatomical area visualized in $R(x)$.

The two knowledge models presented here illustrate the ability to take into consideration some non-trivial morphological and geometric information. It has to be remarked that, by construction, these models can provide information elements which

\footnotetext{
${ }^{1}$ Although $\mathcal{A}^{d}$ and $\mathcal{M}$ can both be expressed in terms of probability maps, they do not have the same semantics. This difference results in particular from the nature of the structures modelled by these fields: a vessel segment and a whole network, respectively.
} 
can be quite accurate, since they are expressed at the same level of details than the resolution of the images from which they are extracted.

Figure 1: Knowledge modelling. First row - Shape model for the portal vein entry, in the liver $(\mathcal{M})$. From left to right: three orthogonal slices (sagittal, coronal and axial). Second row - Statistical atlas $(\mathcal{A})$ of the cerebral vascular network. From left to right: vessel presence probability $\left(\mathcal{A}^{d}\right)$, mean size (part of $\mathcal{A}^{t}$ ), and anatomical reference $(R)$.
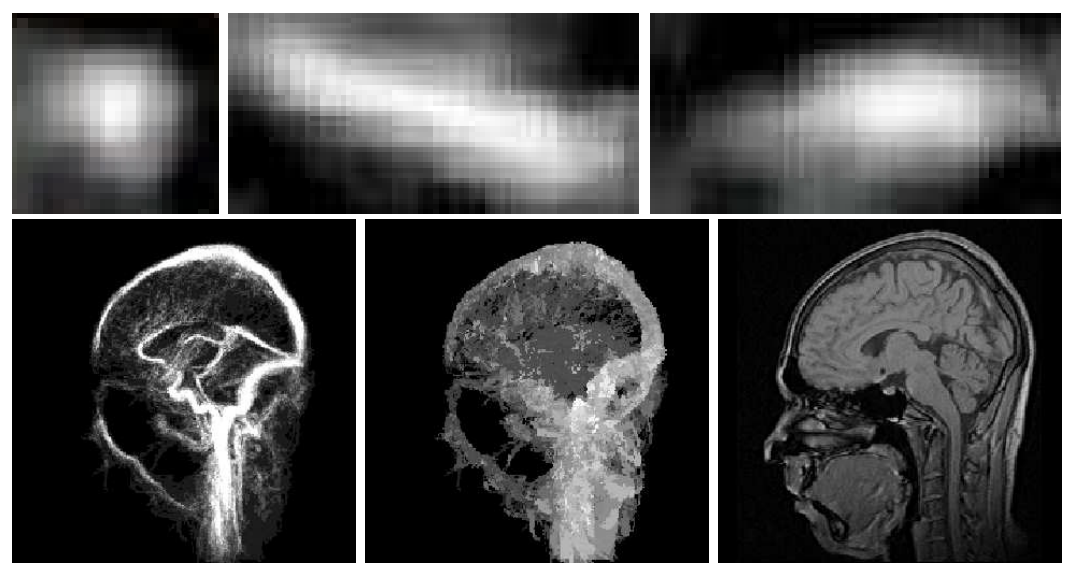

\section{Hit-or-miss transform}

The hit-or-miss transform is an operator enabling to detect some configurations of points satisfying constraints related to two structuring elements [19]. Initially defined for binary images, this operator has been extended to grey-level images under various forms $[16,19,10]$. All these variants of the grey-level hit-or-miss transform can, in a simple way, be unified and expressed as the composition $V \circ D_{A, B}$ of a fitting operator:

$$
\mid \begin{array}{rlc}
D_{A, B}: \mathbb{Z}^{E} & \rightarrow \mathcal{P}(E \times \mathbb{Z}) \\
I & \mapsto \quad\left\{(p, t) \in E \times \mathbb{Z} \mid A_{p, t} \leq I \leq B_{p, t}\right\}
\end{array}
$$

and a valuation operator $V: \mathcal{P}(E \times \mathbb{Z}) \rightarrow \mathbb{Z}^{E}$. Here, $\leq$ is the pointwise order on the functions $E \rightarrow \mathbb{Z}$ while $A, B: E \rightarrow \mathbb{Z}$ are structuring functions ${ }^{2}$ [17, 18]. In fact, as $A_{p, t} \leq I \leq B_{p, t} \Leftrightarrow\left(I \oplus B^{*}\right)(p) \leq t \leq(I \ominus A)(p)$ (where the structuring element $B^{*}$ is defined by $B^{*}(x)=-B(-x)$ ), the computation of the result provided by this operator can be reduced to the computation of an erosion and a dilation. Finally, the purpose of the valuation operator $V$ is to associate to each point of the initial image, detected by $D_{A, B}$, a value expressing a given information; this valuation can have three forms:

\footnotetext{
${ }^{2}$ We mean, by structuring function, a numerical function which represents a grey-level structuring element. Practically, structuring functions are often considered as flat, i.e., as having a constant value (generally 0 ) on their support (noted supp), and $-\infty$ elsewhere.
} 
supremal [16], integral [19] or binary. An illustration of this operator is proposed in Figure 3.

Figure 2: Grey-level hit-or-miss transform. Here, $E=\mathbb{Z}$ and $T=[0 \ldots t] \subset \mathbb{N}$. Two flat structuring elements $A$ and $B$ (where the origin is the left pixel of $A$ ) are used. From top to bottom are illustrated the grey-level hit-or-miss with supremal, integral and binary valuation, respectively. The initial image is depicted with dash lines.

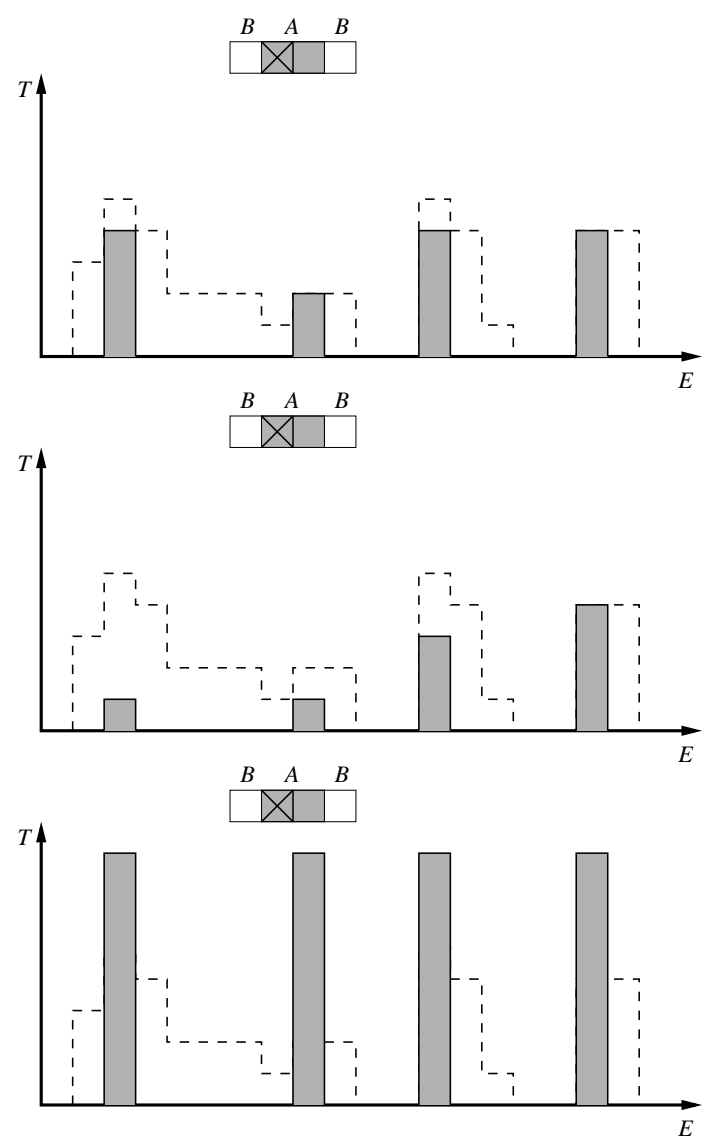

The grey-level hit-or-miss transform is a quite interesting tool in the context of medical image segmentation. Indeed, by definition, it enables to take into account not only photometric information (the structuring elements being grey-level functions), but also morphological and geometric information, thanks to the support of these structuring elements. This kind of information can, moreover, be related either to the objects or their neighbourhood. 


\section{Application: two vessel segmentation examples}

Segmentation of angiographic images is generally a hard issue. This is especially a consequence of the small size and the complex structure of vessels, but also of the specific properties and the quality of the images (resolution and signal to noise ratio, which are generally low), which are then difficult to analyse, even for radiologists. The use of a priori knowledge can enable to overcome these difficulties by taking advantage of the whole available information related to an image and its content. Two examples of segmentation algorithms based on this methodology, and dealing with different kinds of images and vessels, are described hereafter.

Figure 3: Angiographic images. Left: axial slice of an X-ray CT scan, visualizing (on the left part of the image) the hepatic structures and the portal network branches. Right: sagittal slices of a phase-contrast MRA (magnitude and phase images).
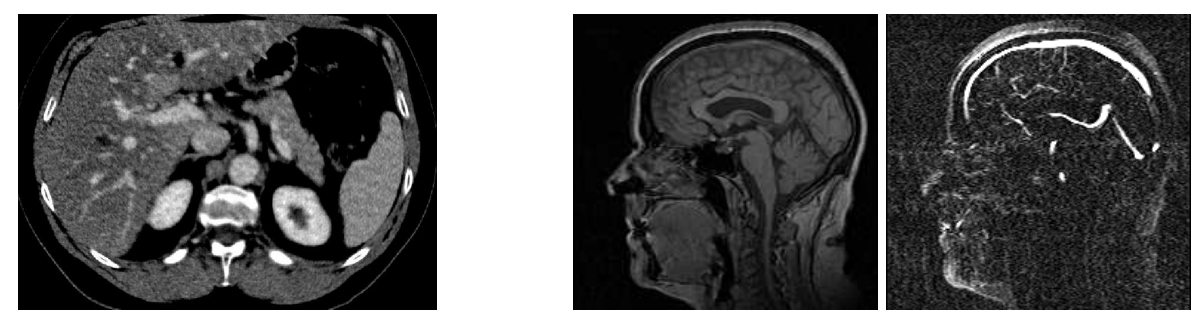

\subsection{Liver vascular network segmentation from X-ray CT-scan}

The liver is composed of two main vascular networks: the portal network and the hepatic network. The segmentation of the portal network from X-ray Computed Tomography scanner (CT-scan) images, such as the one illustrated in Figure 4 (left), is of high importance. Indeed, the anatomical partitioning of the liver into anatomical segments, which is crucial in hepatic surgery, is based on this network.

A portal network segmentation method, proposed in [12], is based on both regiongrowing and hit-or-miss transform. It takes as input a CT-scan angiographic image $I: E \rightarrow \mathbb{Z}$ and a set of structuring elements designed from a set of knowledge related to the portal network (formalized thanks to shape models). It provides as output a binary image ${ }^{3} S: E \rightarrow\{0,1\}$ of the vessels visualized in $I$.

The region-growing algorithms build a partition of the image from one or several seeds by iteratively adding point which verify ad hoc criteria. The approach proposed here consists in defining as seed the portal vein entry in the liver, which constitutes the root of the vascular tree. It is detected in an automatic fashion, by using a combination of grey-level hit-or-miss operators, the structuring elements of which are defined thanks to a shape model $\mathcal{M}$ such as the one described in Section 2. From the set $G$ containing

\footnotetext{
${ }^{3}$ Such binary images are generally considered, by abuse of notation, as sets $S \in \mathcal{P}(E)$ actually corresponding to $S^{-1}(\{1\})$.
} 
the points of the seed, the region-growing process can be defined as the generation of a sequence of sets $\left(S_{k}\right)_{k \in \mathbb{N}}$ where $S_{k} \subseteq E$

$$
\left\{\begin{array}{l}
S_{0}=G \\
S_{k+1}=S_{k} \cup\{x\} \quad \text { if } \exists x \in \Gamma\left(S_{k}\right) \text { such that } C\left(I, S_{k}, x\right)=\text { true }
\end{array}\right.
$$

where $\Gamma\left(S_{k}\right)$ represents the set of the points forming the neighbourhood of the set $S_{k}$, and $C$ if the considered growing criterion. The result of the segmentation is then defined as $S=\lim _{k} S_{k}=\bigcup_{k} S_{k}$.

The region-growing criterion considered here relies on a hit-or-miss transform analysis of the neighbourhood of the considered point $x$, which enables to characterize this point as belonging or not to a tubular structure. This analysis consists in exploring, in $x$, the neighbourhood made of three discrete circles oriented according to the three principal directions. The criterion is satisfied if, for at least one circle, the intensity of all its points is lower than the intensity of the tested point $x$. More formally, we have

$$
C\left(I, S_{k}, x\right)= \begin{cases}\text { true } & \text { if } x \in \Gamma\left(S_{k}\right) \text { and } \max _{i=1}^{3}\left[V \circ D_{O, R_{i}}(I)\right](x)>0 \\ \text { false } & \text { otherwise }\end{cases}
$$

where $O=i_{0,0}\left(i_{p, t}\right.$ representing the impulse function of value $t$ at the point $\left.p\right), R_{i}$ ( $i=1$ to 3 ) are flat structuring functions whose support is formed by three circles being parallel to the axial, coronal and sagittal planes, respectively (and whose radius and thickness of the support, such as the -positive- intensity can be parametrized), and $V$ is the integral valuation operator defined by:

$$
V(X)(x)=|\{t \in \mathbb{Z} \mid(x, t) \in X\}|
$$

This method enables to provide a segmentation of the portal network presenting sound anatomical properties (the generation of a tubular and tree-like structure is made possible by the growing process and the use of coherent structuring elements). Moreover, it can also permit (by assigning, to each segmented point, the value provided by $V$ ) to obtain a "confidence score" related to the photometric properties of the initial image. This method has been applied on a database composed of 16 images, providing results such as the one illustrated in Figure 4.2 (left).

\subsection{Brain vessel segmentation from MRI data}

There exist several kinds of angiographic MRI data (also called magnetic resonance angiography -MRA). Among them, one of the most frequently used is phase-contrast MRA, illustrated in Figure 4 (right). It presents the particularity to provide a bimodal image (i.e. a couple of images, each one acquired according to a specific modality), composed of a magnitude image, visualizing the morphological structures, and a phase image, visualizing the flowing blood.

A segmentation method proposed in [15] consists in segmenting the MRA phase images by performing a filtering based on grey-level hit-or-miss, the structuring elements of which are designed according to the tubular structure of the vessels. These structuring elements are composed by a discrete sphere, for the object, and a sampled 
discrete circle having the same centre as the sphere, for the background. Such a definition leads to a family of structuring elements induced by the degrees of freedom (size, orientation) of the model. The purpose of the method is to choose the elements to apply according to the position in the image, in order to obtain results being similar to those which would be computed by an exhaustive approach, but with a significantly lower algorithmic/computational cost. The guidance of the method is based on an atlas and the information provided by the magnitude image associated to the phase image to be segmented.

The method takes as input a MRA composed of a magnitude image and a phase image $I_{m}, I_{p}: E \rightarrow \mathbb{Z}$, an atlas $\mathcal{A}$ such as proposed in Section 2, and its anatomical reference $R$. It provides as output a binary image $S: E \rightarrow\{0,1\}$ of the vessels visualized in $I_{p}$. First, a non-rigid registration of $R$ onto $I_{m}$ is performed, in order to obtain a deformation field $\mathcal{D}: E \rightarrow E$ establishing a correspondence between the anatomical structures in $I_{m}$ and $R$. Once this deformation field is obtained, a new atlas $\mathcal{A}^{\prime}=\mathcal{D} \circ \mathcal{A}$ correctly fitted to the anatomical structures of $I$ can be deduced. At each point $x \in E$, a set $E S_{x}$ of structuring elements is then defined as:

$$
E S_{x}= \begin{cases}\left\{(A, B) \mid r_{A}, r_{B}, \theta_{B}, \phi_{B} \text { coherent with } \mathcal{A}^{\prime t}(x), \mathcal{A}^{\prime o}(x)\right\} & \text { if } \mathcal{A}^{\prime d}(x)>0 \\ \emptyset & \text { otherwise }\end{cases}
$$

where $\operatorname{supp}(A)$ is a discrete sphere of radius $r_{A}$, and $\operatorname{supp}(B)$ is a sampled concentric discrete circle of radius $r_{B}$ of orientation $\left(\theta_{B}, \phi_{B}\right)$. Here, the elements $A$ and $B$ are functions having constant values $a$ and $b$, then we set $a-b=1$ in order to give to the geometric information a stronger influence than the one of photometric information (which is quite unreliable in this kind of images). Then, for all $x \in E$ and all $(A, B) \in$ $E S_{x}$, a grey-level hit-or-miss transform is applied to determine whether $x$ corresponds to a vessel, w.r.t. the anatomical knowledge at this position. If $x$ is evaluated as being vascular, its dilation by the element $A$ involved in its detection is added to the result image $S$. More concretely, $S$ is defined as:

$$
S=\bigcup\left\{A \oplus\{x\} \mid \exists(A, B) \in S E_{x},\left[V \circ D_{A, B}\left(I_{p}\right)\right](x)>0\right\}
$$

where $V$ is defined as in Section 4.1. This method has been applied on a database of 15 images, providing results such as the ones illustrated in Figure 4.2 (right).

\section{Conclusion}

The integration of high-level knowledge in segmentation methods, and more generally in medical image processing ones, constitutes a recent and extremely promising way towards the design of radiology-assistance tools actually usable in clinical routine. In this context, mathematical morphology proposes a methodological framework perfectly fitted, especially thanks to its ability to take into consideration geometric and morphological properties which naturally formalize several anatomical knowledge elements.

This chapter has intended to illustrate this assertion by presenting few concepts and methods (the relevance of which has been proved by validations carried out by 
Figure 4: Segmentation results. Left: method described in Section 4.1 (liver vascular network). Right: method described in Section 4.2 (cerebral vascular network).
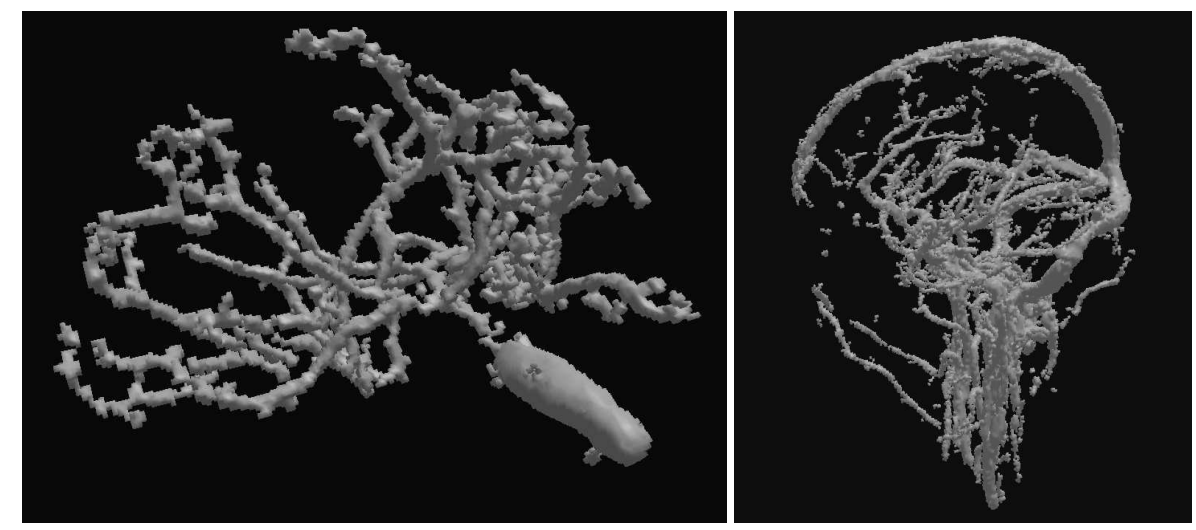

medical experts), which will be -hopefully- sufficiently representative to convince the reader. Several other contributions could actually extend and complete this chapter. The readers who are interested in other works related to mathematical morphologybased segmentation methods of 3D angiographic data can also refer to the following articles $[21,2,20,1]$. More generally, besides the use of morphological and geometric knowledge $[9,13]$, other solutions dealing with the integration of topological knowledge $[6,5,7]$, relational knowledge [3, 8], or even the use of temporal information [4] (see also the previous chapter of this book) for the segmentation of medical images, have been investigated during the last years, and have already led to quite interesting results.

\section{References}

[1] B. Bouraoui, C. Ronse, J. Baruthio, N. Passat, and P. Germain. 3D segmentation of coronary arteries based on advanced Mathematical Morphology techniques. Computerized Medical Imaging and Graphics, pages -, 2010.

[2] B. Caldairou, B. Naegel, and N. Passat. Segmentation of complex images based on component-trees: Methodological tools. In M.H.F. Wilkinson and J.B.T.M. Roerdink, editors, International Symposium on Mathematical Morphology - ISMM'09, 9th International Symposium, Proceedings, volume 5720 of Lecture Notes in Computer Science, pages 171-180, Groningen, The Netherlands, August 24-27 2009. Springer.

[3] O. Colliot, O. Camara, and I. Bloch. Integration of fuzzy spatial relations in deformable models - application to brain MRI segmentation. Pattern Recognition, 39(8):1401-1414, August 2006. 
[4] J. Cousty, L. Najman, M. Couprie, S. Clément-Guinaudeau, T. Goissen, and J. Garot. Segmentation of 4D cardiac MRI: automated method based on spatiotemporal watershed cuts. Image and Vision Computing, $\mathrm{xx}(\mathrm{xx}): \mathrm{xxx}-\mathrm{xxx}, \mathrm{xxxxx}$ 2010.

[5] X. Daragon and M. Couprie. Segmentation topologique du néo-cortex cérébral depuis des données IRM dans le cadre de la topologie des ordres. In Reconnaissance des Formes et Intelligence Artificielle - RFIA'02, 13e Congrès Francophone AFRIF-AFIA, Proceedings, volume 3, pages 809-818, Angers, France, January 8-10 2002.

[6] P. Dokládal, C. Lohou, L. Perroton, and G. Bertrand. Liver blood vessels extraction by a 3-D topological approach. In C. Taylor and A.C.F. Colchester, editors, Medical Image Computing and Computer-Assisted Intervention - MICCAI'99, 2nd International Conference, Proceedings, volume 1679 of Lecture Notes in Computer Science, pages 98-105, Cambridge, United Kingdom, September 1922 1999. Springer.

[7] S. Faisan, N. Passat, V. Noblet, R. Chabrier, and C. Meyer. Topology preserving warping of binary images: Application to atlas-based skull segmentation. In D.N. Metaxas, L. Axel, G. Fichtinger, and G. Székely, editors, Medical Image Computing and Computer-Assisted Intervention - MICCAI'08, 11 th International Conference, Proceedings, Part I, volume 5241 of Lecture Notes in Computer Science, pages 211-218, New York, NY, USA, September, 6-10 2008. Springer.

[8] A. Moreno, C. M. Takemura, O. Colliot, O. Camara, and I. Bloch. Using anatomical knowledge expressed as fuzzy constraints to segment the heart in CT images,. Pattern Recognition, 41(8):2525-2540, August 2008.

[9] B. Naegel. Using mathematical morphology for the anatomical labeling of vertebrae from 3D CT-scan images. Computerized Medical Imaging and Graphics, 31(3):141-156, 2007.

[10] B. Naegel, N. Passat, and C. Ronse. Grey-level hit-or-miss transforms - Part I: Unified theory. Pattern Recognition, 40(2):635-647, 2007.

[11] B. Naegel, N. Passat, and C. Ronse. Grey-level hit-or-miss transforms- Part II : Application to angiographic image processing. Pattern Recognition, 40(2):648658, 2007.

[12] B. Naegel, C. Ronse, and L. Soler. Using grey-scale hit-or-miss transform for segmenting the portal network of the liver. In C. Ronse, L. Najman, and É. Decencire, editors, Mathematical Morphology: 40 years on. Proceedings of the 7th International Symposium on Mathematical Morphology, volume 30 of Computational Imaging and Vision, pages 429-440. Springer SBM, 2005.

[13] N. Passat, C. Ronse, J. Baruthio, J.-P. Armspach, and J. Foucher. Watershed and multimodal data for vessel segmentation: Application to the superior sagittal sinus. Image and Vision Computing, 25(4):512-521, 2007. 
[14] N. Passat, C. Ronse, J. Baruthio, J.-P. Armspach, and C. Maillot. Cerebral vascular atlas generation for anatomical knowledge modeling and segmentation purpose. In C. Schmid, S. Soatto, and C. Tomasi, editors, IEEE CVPR'05, Proceedings, volume 2, pages 331-337, 2005.

[15] N. Passat, C. Ronse, J. Baruthio, J.-P. Armspach, and C. Maillot. Magnetic resonance angiography: From anatomical knowledge modeling to vessel segmentation. Medical Image Analysis, 10(2):259-274, 2006.

[16] C. Ronse. A lattice-theoretical morphological view on template extraction in images. Journal of Visual Communication and Image Representation, 7(3):273295, 1996.

[17] J. Serra. Image Analysis and Mathematical Morphology. Academic Press, London, 1982.

[18] J. Serra, editor. Image Analysis and Mathematical Morphology. II: Theoretical Advances. Academic Press, London, 1988.

[19] P. Soille. Morphological Image Analysis: Principles and Applications. SpringerVerlag Berlin Heidelberg, 2nd edition, 2003.

[20] O. Tankyevych, H. Talbot, P. Dokládal, and N. Passat. Direction-adaptive greylevel morphology. application to $3 \mathrm{D}$ vascular brain imaging. In International Conference on Image Processing - ICIP'09, 16th International Conference, Proceedings, pages 2261-2264, Cairo, Egypt, November 7-11 2009. IEEE Signal Processing Society.

[21] M.H.F. Wilkinson and M.A. Westenberg. Shape preserving filament enhancement filtering. In W.J. Niessen and M.A. Viergever, editors, Medical Image Computing and Computer-Assisted Intervention - MICCAI 2001, 4th International Conference, Proceedings, volume 2208 of Lecture Notes in Computer Science, pages 770-777, Utrecht, The Netherlands, October 14-17 2001. Springer. 